\title{
4D Atomic Electron Tomography
}

Jianwei Miao ${ }^{1 *}$, Jihan Zhou ${ }^{1}$, Yongsoo Yang ${ }^{1}$, Yao Yang ${ }^{1}$, Dennis S. Kim ${ }^{1}$, Andrew Yuan ${ }^{1}$, Xuezeng Tian $^{1}$, Colin Ophus², Fan Sun ${ }^{3}$, Andreas K. Schmid², Michael Nathanson ${ }^{4}$, Hendrik Heinz ${ }^{4}$, Qi An ${ }^{5}$, Hao Zeng $^{3}$ and Peter Ercius ${ }^{2}$

1. Department of Physics \& Astronomy and California NanoSystems Institute, University of California, Los Angeles, CA, USA.

2. National Center for Electron Microscopy, Molecular Foundry, Lawrence Berkeley National

Laboratory, Berkeley, CA, USA.

3. Department of Physics, University at Buffalo, the State University of New York, Buffalo, NY, USA.

4. Department of Chemical and Biological Engineering, University of Colorado at Boulder, Boulder, CO, USA.

5. Chemical and Materials Engineering, University of Nevada, Reno, NV, USA.

* Corresponding author: miao@physics.ucla.edu

Perfect crystals are rare in nature. Real materials often contain crystal defects such as grain boundaries, dislocations, stacking faults, interfaces, surface reconstructions and point defects. These crystal defects and their dynamics strongly affect material properties and functionality. A major challenge in materials characterization is to determine the $3 \mathrm{D}$ atomic positions of crystal defects and monitor their dynamics. To tackle this challenge, we have developed atomic electron tomography (AET) to image the 3D crystal defects in materials at atomic resolution [1-4] using powerful computational algorithms [5, 6] More recently, we determined the 3D atomic arrangement of chemical order/disorder in an FePt nanoparticle with $22 \mathrm{pm}$ precision [7]. The measured atomic positions and chemical species were used as direct input to quantum mechanical calculations to correlate crystal defects and chemical order/disorder with material properties at the single-atom level [7]. Moreover, using FePt nanoparticles as a model system, we advanced AET to study nucleation at 4D atomic resolution [8]. We revealed that early stage nuclei are irregularly shaped, each has a core of one to few atoms with the maximum order parameter, and the order parameter gradient points from the core to the boundary of the nucleus. We captured the structure and dynamics of the same nuclei undergoing growth, fluctuation, dissolution, merging and/or division, which are regulated by the order parameter distribution and its gradient. These experimental observations differ from classical nucleation theory (CNT) and to explain them we proposed the order parameter gradient (OPG) model [8]. We showed the OPG model generalizes CNT and energetically favours diffuse interfaces for small nuclei and sharp interfaces for large nuclei. We further corroborated this model using molecular dynamics simulations of heterogeneous and homogeneous nucleation in liquid-solid phase transitions of Pt. We anticipate that the OPG model is applicable to different nucleation processes and our experimental method opens the door to study the structure and dynamics of materials with 4D atomic resolution [9].

References:

[1] J Miao, P Ercius and SJL Billinge, Science 353 (2016), p. aaf2157.

[2] MC Scott et al., Nature 483 (2012), p. 444.

[3] CC Chen et al., Nature 496 (2013), p. 74.

[4] R Xu et al., Nature Mater. 14 (2015), p. 1099.

[5] J Miao, F Föster and O Levi, Phys. Rev. B 72 (2005), p. 052103. 
[6] A Pryor Jr. et al., GENFIRE: Sci. Rep. 7 (2017), p. 10409.

[7] Y Yang et al., Nature 542 (2017), p. 75.

[8] J Zhou et al., arXiv:1807.10709 (2018).

[9] This work was supported by STROBE: A National Science Foundation Science \& Technology

Center under Grant No. DMR 1548924, the Office of Basic Energy Sciences of the U.S. DOE (Grant

No. DE-SC0010378) and the NSF DMREF program (DMR-1437263). ADF-STEM imaging was performed on TEAM I at the Molecular Foundry, which is supported by the Office of Science, Office of Basic Energy Sciences of the U.S. Department of Energy under Contract No. DE-AC02-05CH11231.
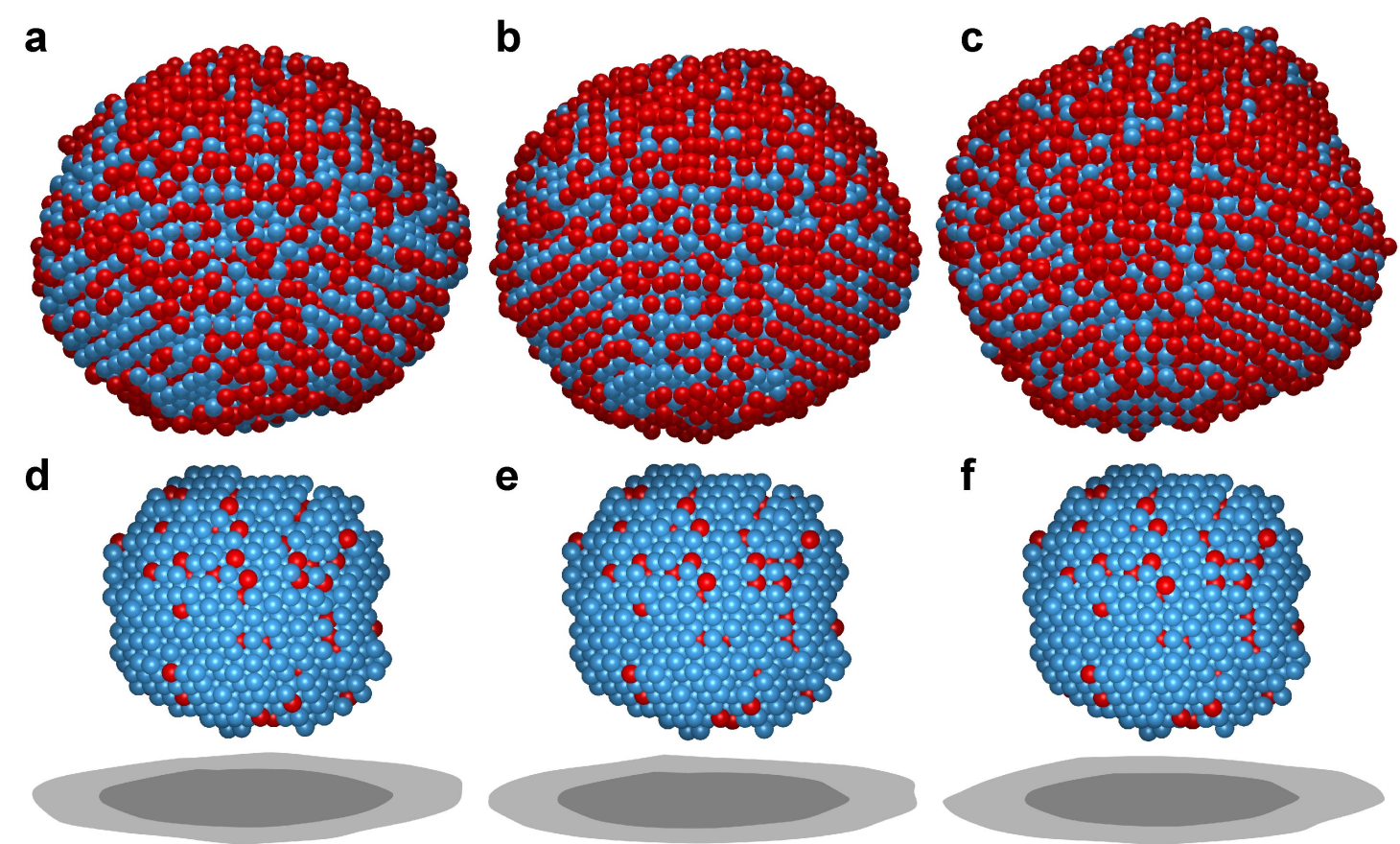

e
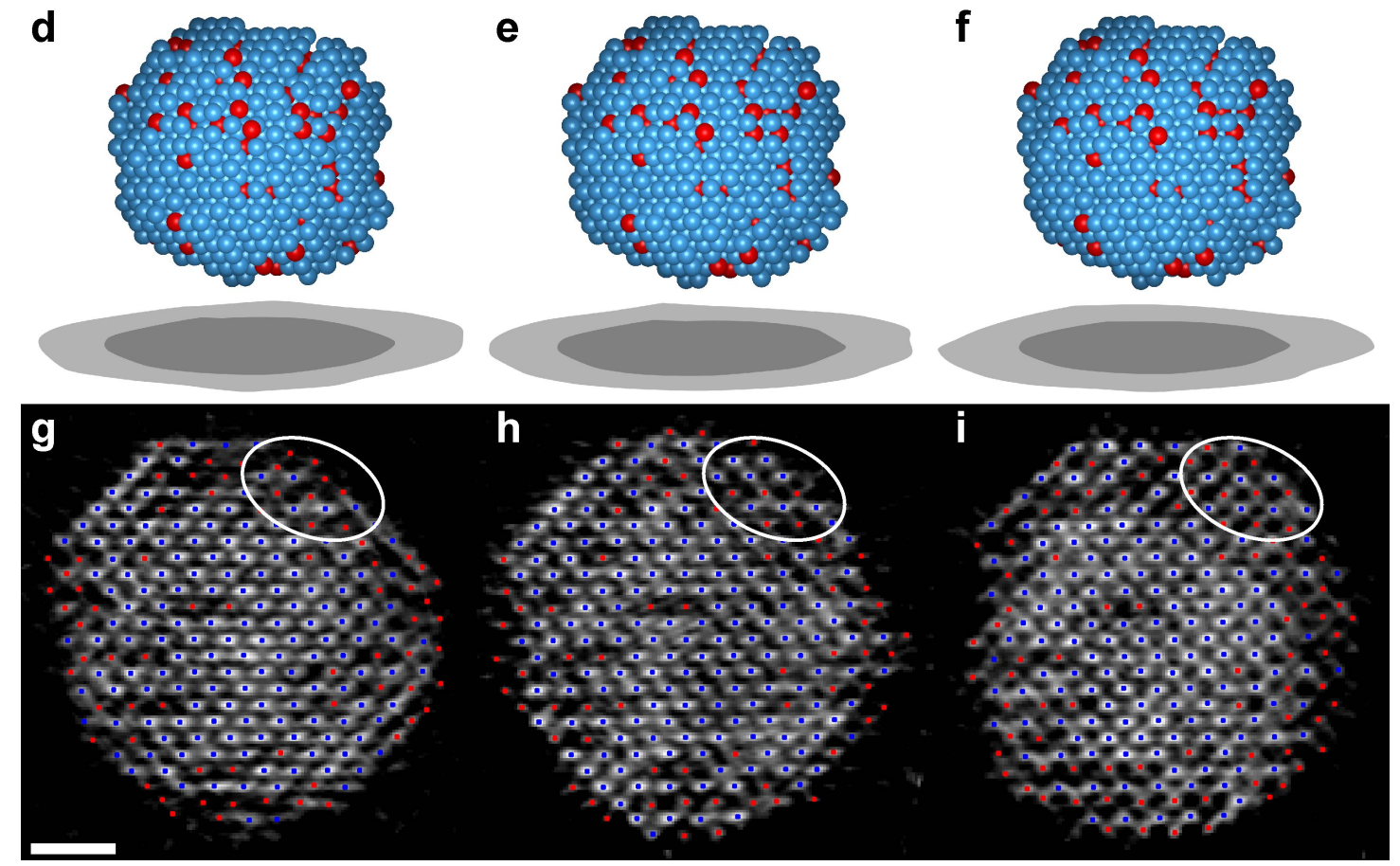

Figure 1. Capturing 4D atomic motion with AET. a-c, 3D atomic models (Fe in red and Pt in blue) of an FePt nanoparticle with an accumulated annealing time of 9, 16 and 26 minutes, respectively. The 3D shape of the nanoparticle was similar from 9 to 16 minutes, but changed from 16 to 26 minutes. d-f, The Pt-rich core of the nanoparticle remained the same for the three annealing times. The light and dark grey projections show the whole nanoparticle and the core, respectively. g-i, The same internal atomic layer of the nanoparticle along the [010] direction at the three annealing times (Fe in red and Pt in blue), where a fraction of the surface and sub-surface atoms were re-arranged to form $\mathrm{L} 1_{0}$ phases (ellipses), but the Pt-rich core of the nanoparticle remained the same. 